\title{
COMPLEX SPECIALIZATIONS OF THE REDUCED GASSNER REPRESENTATION OF THE PURE BRAID GROUP
}

\author{
MOHAMMAD N. ABDULRAHIM
}

(Communicated by Ronald M. Solomon)

\begin{abstract}
We will give a necessary and sufficient condition for the specialization of the reduced Gassner representation $G_{n}(z): P_{n} \rightarrow G L_{n-1}(\mathbb{C})$ to be irreducible. It will be shown that for $z=\left(z_{1}, \ldots, z_{n}\right) \in\left(\mathbb{C}^{*}\right)^{n}, G_{n}(z)$ is irreducible if and only if $z_{1} \ldots z_{n} \neq 1$.
\end{abstract}

\section{INTRODUCTION}

In this paper, we will give a necessary and sufficient condition for the complex specialization of the reduced Gassner representation of the pure braid group, $P_{n}$, to be irreducible. The argument that will be presented here is purely algebraic.

In section 1, we will state a theorem that gives a classification, up to equivalence, of all irreducible complex representations of a free group of a certain rank, for which the image of each generator is a pseudoreflection. Then, as an application, we state a necessary and sufficient condition for the irreducibility of the complex specialization of the Burau representation of the braid group, $B_{n}$. This was proved in $[4, \mathrm{p} .9]$. In section 2, we will define, up to equivalence, the Gassner representation of $P_{n}$ and show that it is reducible to an $n-1$ representation called the reduced Gassner representation which turns out to be irreducible in $G L_{n-1}\left(\mathbb{Z}\left[y_{1}^{ \pm 1}, \ldots, y_{n}^{ \pm 1}\right]\right)$. To apply Theorem 2, which is stated in section 1, for the complex specialization of the reduced Gassner representation of $P_{n}$, we construct a certain normal free subgroup of $P_{n}$ of rank $n-1$; this will be done in section 3 and will be used as a tool to prove our main theorem in section 4 .

Main Theorem. Let $G_{n}(z): P_{n} \rightarrow G L_{n-1}(\mathbb{C})$ be the reduced Gassner representation obtained by the specialization: $y_{i} \rightarrow z_{i}$, where $z=\left(z_{1}, \ldots, z_{n}\right) \in\left(\mathbb{C}^{*}\right)^{n}$ and the $y_{i}$ 's are the independent indeterminates used in defining this representation. Then $G_{n}(z)$ is irreducible if and only if $z_{1} \ldots z_{n} \neq 1$.

\section{Complex specializations of the Reduced Burau Representation}

Definition 1. Let $\mathbb{C}^{r}=r \times 1$ (or column) vectors, $\overline{\mathbb{C}}^{r}=1 \times r$ (or row) vectors. A matrix $X \in M_{r}(\mathbb{C})$ is a pseudoreflection if $X-I$ has rank 1 . We regard $M_{r}(\mathbb{C})$ as

Received by the editors December 23, 1995.

1991 Mathematics Subject Classification. Primary 20F36.

Key words and phrases. Braid group, pure braid group, Burau representation, Gassner representation.

The results in this paper were written under the direction of Professor Edward Formanek whose help and encouragement are greatly appreciated. 
acting from the left on column vectors so that eigenvectors and invariant subspaces lie in $\mathbb{C}^{r}$. If $X$ is a pseudoreflection, then

$$
X=I-A B,
$$

where $A \in \mathbb{C}^{r}$ and $B \in \overline{\mathbb{C}^{r}}$. This representation is unique, except that if $a \in \mathbb{C}^{*}$, then $X=I-A B=I-(a A)\left(a^{-1} B\right)$. The eigenvalues of $X$ are 1 , with multiplicity $r-1$, and $1-B A$ with multiplicity 1 . Hence $X$ is invertible if and only if $B A \neq 1$.

We say $G$ is an irreducible subgroup of $G L_{r}(\mathbb{C})$ if it spans $G L_{r}(\mathbb{C})$ as a vectorspace over $\mathbb{C}$. To know the connection between "irreducible subgroup" and "irreducible representation of a group", see [3, p.167].

The following theorem gives a criterion for the group generated by $r$ pseudoreflections in $G L_{r}(\mathbb{C})$ to be irreducible. We will use this result to find conditions for the Burau and Gassner representations in $G L_{n}(\mathbb{C})$ to be irreducible.

Theorem 2 ([4, p.6]). Let $X_{1}=I-A_{1} B_{1}, \ldots, X_{r}=I-A_{r} B_{r}$ be $r$ invertible pseudoreflections in $M_{r}(\mathbb{C})$, where $r \geq 2$. Let $\tau$ be the directed graph whose vertices are $1,2, \ldots, r$, and which has a directed edge from $i$ to $j(i \neq j)$ precisely when $B_{i} A_{j} \neq 0$. Let $G$ be the subgroup of $G L_{r}(\mathbb{C})$ generated by $X_{1}, \ldots, X_{r}$. Then

(a) The following are equivalent.

(1) $G$ is an irreducible subgroup of $G L_{r}(\mathbb{C})$.

(2) For each $i \neq j, 1 \leq i, j \leq r$, the graph $\tau$ contains a directed path from $i$ to $j$, $\left\{A_{1}, \ldots, A_{r}\right\}$ is a basis for $\mathbb{C}^{r}$, and $\left\{B_{1}, \ldots, B_{r}\right\}$ is a basis for $\overline{\mathbb{C}}^{r}$.

(3) For each $i \neq j, 1 \leq i, j \leq r$, the graph $\tau$ contains a directed path from $i$ to $j$, and $\left(B_{i} A_{j}\right) \in M_{r}(\mathbb{C})$ is invertible. $\left(\left(B_{i} A_{j}\right)\right.$ is the matrix whose entries are $B_{i} A_{j}$ for different values of $i$ and $j$.)

(b) Suppose that $G=\left\langle X_{1}, \ldots, X_{r}\right\rangle$ and $H=\left\langle Y_{1}, \ldots, Y_{r}\right\rangle$ are irreducible subgroups of $G L_{r}(\mathbb{C})$ generated by pseudoreflections $X_{i}=I-A_{i} B_{i}$ and $Y_{i}=I-C_{i} D_{i}$. Then there is a matrix $T \in G L_{r}(\mathbb{C})$ such that $T X_{i} T^{-1}=Y_{i}$ for $i=1, \ldots, r \Longleftrightarrow$ there exist $a_{1}, \ldots, a_{r} \in \mathbb{C}^{*}$ such that $D_{i} C_{j}=a_{i}^{-1} a_{j} B_{i} A_{j}$, i.e. $\left(B_{i} A_{j}\right)$ and $\left(D_{i} C_{j}\right)$ are congugate by a diagonal matrix.

(c) If $G=\left\langle X_{1}, \ldots, X_{r}\right\rangle$ is an irreducible subgroup of $G L_{r}(\mathbb{C})$, generated by pseudoreflections $X_{1}, \ldots, X_{r}$, then there is a matrix $T \in G L_{r}(\mathbb{C})$ such that $T X_{i} T^{-1}=$ $I-P_{i} Q_{i}$ where $Q_{i}=(0,0, \ldots, 1,0, \ldots, 0)$, the $i$-th standard basis vector of $\overline{\mathbb{C}}^{r}$.

Part (b) of Theorem 2 is a classification, up to equivalence, of all irreducible representations

$$
F_{r}\left\langle x_{1}, \ldots, x_{r}\right\rangle \rightarrow G L_{r}(\mathbb{C}),
$$

where $F_{r}\left\langle x_{1}, \ldots, x_{r}\right\rangle$ is a free group of rank $r$, for which the image of each $x_{i}$ is a pseudoreflection. Although this is a rather restricted class of representations, it includes the reduced Burau representation and the restriction of the reduced Gassner representation of the pure braid group to a certain normal free subgroup, the normal closure of the square of one of the standard generators of the braid group. We will now show that the images of the generators of $B_{n}$, namely, $\sigma_{1}, \ldots, \sigma_{n-1}$, under the complex specialization of the reduced Burau representation are pseudoreflections. Here $B_{n}$ denotes the braid group on $n$ strings. We will deal with $B_{n}$ as an abstract group with generators and relations. For more details, see [5, p.7] and [2, p.118]. Then we will apply Theorem 2 to specializations of the reduced Burau 
representation

$$
\overline{\beta_{n}}(z): B_{n} \rightarrow G L_{n-1}(\mathbb{C}) .
$$

Then $\overline{\beta_{n}}(z)=I-A_{i} B_{i}$, where

$$
\begin{aligned}
& A_{1}=\left(\begin{array}{llll}
z+1 & 1 & 0 \ldots & 0
\end{array}\right)^{T}, \\
& A_{i}=\left(\begin{array}{lllll}
\underbrace{0 \ldots 0}_{i-2} & z & z+1 & 1 & \underbrace{0 \ldots 0}_{n-i-2}
\end{array}\right)^{T},
\end{aligned}
$$

$i=2, \ldots, n-2$, and

$$
A_{n-1}=\left(\begin{array}{lllll}
0 & 0 \ldots & 0 & z & z+1
\end{array}\right)^{T},
$$

where $T$ is the transpose, and $\left\{B_{1}, \ldots, B_{n-1}\right\}$ is the standard basis of $\overline{\mathbb{C}}^{n-1}$. The associated matrix $\left(B_{i} A_{j}\right)$ is

$$
\left(\begin{array}{cccccc}
z+1 & z & 0 & \ldots & 0 & 0 \\
1 & z+1 & z & \ldots & 0 & 0 \\
0 & 1 & z+1 & \ldots & 0 & 0 \\
0 & 0 & 1 & \ldots & 0 & 0 \\
\vdots & \vdots & \vdots & & \vdots & \vdots \\
0 & 0 & 0 & \ldots & z+1 & z \\
0 & 0 & 0 & \ldots & 1 & z+1
\end{array}\right)
$$

Lemma $3\left(\left[4\right.\right.$, p.9]). For $z \in \mathbb{C}^{*}, \overline{\beta_{n}}(z): B_{n} \rightarrow G L_{n-1}(\mathbb{C})$ is irreducible if and only if $z$ is not a root of $f_{n}(t)=t^{n-1}+t^{n-2}+\ldots+t+1$.

Proof. A simple induction shows that $\operatorname{det}\left(B_{i} A_{j}\right)=z^{n-1}+z^{n-2}+\ldots+z+1$. Then by Theorem 2 , the result follows.

\section{The Gassner Representation of $P_{n}$}

The pure braid group, $P_{n}$, is defined as the kernel of the homomorphism $B_{n} \rightarrow S_{n}$ defined by $\sigma_{i} \rightarrow(i, i+1), \quad 1 \leq i \leq n-1$. It admits a presentation with generators

$$
A_{i j}=\sigma_{j-1} \sigma_{j-2} \ldots \sigma_{i+1} \sigma_{i}^{2} \sigma_{i+1}^{-1} \ldots \sigma_{j-2}^{-1} \sigma_{j-1}^{-1} ; \quad 1 \leq i<j \leq n .
$$

These generators have relations among them. For more details, see [5, pp.19-21]. The Gassner representation of $P_{n}$, denoted by $\gamma_{n}$, is defined explicitly in $[1, \mathrm{p} .15]$ and $[2, \mathrm{p} .119]$. Then it is clear that the image of $A_{i j}{ }^{-1}$ under $\gamma_{n}$ is a pseudoreflection $X_{i j}$ where $X_{i j}=I-P_{i j} Q_{i j}$, and $P_{i j}$ is the column vector defined by

$$
(\underbrace{0 \ldots 0}_{i-1} y_{j}-1 \quad\left(y_{i}-1\right)\left(1-y_{j}\right) \ldots \quad\left(y_{i}-1\right)\left(1-y_{j}\right) \quad\left(y_{i}-1\right)\left(-y_{j}\right) \quad \underbrace{0 \ldots 0}_{n-j})^{T}
$$

and

$$
Q_{i j}=(\underbrace{0 \ldots 0}_{i-1}-1 \quad 0 \ldots \quad 0 \quad 1 \underbrace{0 \ldots 0}_{n-j}) .
$$

Here $T$ is the transpose. The image of $A_{i j}{ }^{-1}$ under $\gamma_{n}$ was computed rather than the image of $A_{i j}$ for the reason of getting a more convenient representation, in the sense that the image of $A_{i j}{ }^{-1}$ involves only the indices $i$ and $j$. It is then clear that the Gassner representation is reducible because there is an invariant subspace 
spanned by the vector $(1, \ldots, 1)^{T}$, which is fixed by the action of this representation ( $T$ is the transpose). It is common knowledge that the Gassner representation is reducible as was shown in $[2, \mathrm{p} .121]$. We will show that $\gamma_{n}$ is the direct sum of a trivial representation and an irreducible representation called the reduced Gassner representation and denoted by $\overline{\gamma_{n}}$. Notice that for $j \neq n$, the last row of $X_{i j}$ is $(0, \ldots, 0,1)$. Delete the last row and column to obtain an $n-1 \times n-1$ matrix $Y_{i j}$, where $Y_{i j}=I-\overline{P_{i j}} \overline{Q_{i j}}$. Here $\overline{P_{i j}}$ and $\overline{Q_{i j}}$ are the same as $P_{i j}$ and $Q_{i j}$ after deleting one zero from the last row of $P_{i j}$, and one zero from the last column of $Q_{i j}$, respectively. For $j=n$, put $Y_{i n}=I-\overline{P_{i n}} \overline{Q_{i n}}$, where

$$
\overline{P_{i n}}=(\underbrace{\left(1-y_{i}\right) t \ldots\left(1-y_{i}\right) t}_{i-1} \quad 1-y_{i} t \underbrace{1-y_{i} \ldots 1-y_{i}}_{n-1-i})^{T}
$$

and

$$
\overline{Q_{i n}}=\left(\begin{array}{lll}
\underbrace{0 \ldots 0}_{i-1} & 1 & \underbrace{0 \ldots 0}_{n-1-i}
\end{array}\right),
$$

where $t=y_{n}$. By part (b) of Theorem 2, this is a choice for $\overline{P_{i n}}$ and $\overline{Q_{i n}}$ for which the matrix given by the inner product $\left(\overline{Q_{i n}} \overline{P_{j n}}\right)$ is equal to the matrix $\left(Q_{i n} P_{j n}\right)$. The details are found in [1, p.27] and [4, p.6]. Here $Y_{i j}$ is the image of $A_{i j}^{-1}$ under the reduced Gassner representation $\overline{\gamma_{n}}$. There is no explicit representation which is agreed to be "the" reduced Gassner representation of $P_{n}$.

Theorem 4. Let $G$ be a group and $\rho: G \rightarrow G L_{n}(\mathbb{C})$ a representation of degree $n$. Then $\rho$ is a direct sum of a representation of degree $n-1$ and a trivial representation if and only if there are a row vector $w$ and a column vector $v$ fixed by $\rho(G)$ such that $w v=1$.

Proof. Here $G L_{n}(\mathbb{C})$ is considered as acting from the left on column vectors or acting from the right on row vectors.

$\Longrightarrow$ Without loss of generality, let

$$
\rho(x)=\left(\begin{array}{cc}
\bar{\rho}(x) & 0 \\
0 & 1
\end{array}\right)
$$

where $x \in G$. Then it is clear that the vectors $e_{n}$ and $f_{n}$ are fixed by $\rho(G)$, where $e_{n}=(0 \ldots 1), f_{n}=(0 \ldots 1)^{T}$ and $e_{n} f_{n}=1$ ( $T$ is the transpose).

$\Longleftarrow$ Assume that there exist such row vectors and column vectors $w$ and $v$ fixed by $\rho(G)$ and $w v=1$. Let $M$ be an $n \times n$ invertible matrix such that $w M=e_{n}$, then

$$
1=w v=e_{n} M^{-1} v
$$

This implies that $M^{-1} v$ is a column vector of the form $(*, \ldots, *, 1)^{T}$, where the $*$ 's are some complex numbers. Now there exists an invertible matrix $N$ such that $e_{n} N=e_{n}$ and $N f_{n}=M^{-1} v$. To see this, we can choose $N$ as the following matrix:

$$
N=\left(\begin{array}{ccccc}
1 & 0 & \ldots & 0 & * \\
0 & 1 & \ldots & & * \\
\vdots & & \ddots & & \vdots \\
& & & 1 & * \\
0 & \ldots & & 0 & 1
\end{array}\right)
$$


where the last column of $N$ is exactly the vector $M^{-1} v$. Then we get

$$
(w) M N=e_{n} \text { and } M N\left(f_{n}\right)=v .
$$

Consider the equivalent representation of $\rho$, namely $(M N)^{-1} \rho(M N)$; then for $x \in$ $G$, we have

$$
(M N)^{-1} \rho(x)(M N)\left(f_{n}\right)=f_{n} \quad \text { and } \quad\left(e_{n}\right)(M N)^{-1} \rho(x)(M N)=e_{n} .
$$

It is then easy to observe that $(M N)^{-1} \rho(x)(M N)$ must be of the form

$$
\left(\begin{array}{cc}
\bar{\rho}(x) & 0 \\
0 & 1
\end{array}\right)
$$

where $\bar{\rho}$ is a representation of degree $n-1$.

Lemma 5. The Gassner representation $\gamma_{n}$ is the direct sum of the reduced representation of degree $n-1$, denoted by $\overline{\gamma_{n}}$, and the trivial representation.

Proof. In light of Theorem 4, it suffices to find a row vector and a column vector fixed by $\gamma_{n}$ whose inner product is one. Let $w$ be the row vector defined as follows:

$$
w=\frac{1}{y_{1} \ldots y_{n}-1}\left[y_{2} \ldots y_{n}\left(y_{1}-1\right), y_{3} \ldots y_{n}\left(y_{2}-1\right), \ldots, y_{n}\left(y_{n-1}-1\right), y_{n}-1\right]
$$

and

$$
v=(1, \ldots, 1)^{T} .
$$

It is then clear, by direct calculation, that these vectors are fixed by $\gamma_{n}$.

\section{The Restriction of the Reduced GAssner Representation TO A CERTAIN NORMAL FREE SUBGROUP}

To apply Theorem 2 for the reduced Gassner representation of $P_{n}$, we will construct for each $i=1, \ldots, n$ a free normal subgroup of rank $n-1$, namely, $V_{i}$. Let $V_{i}$ be the subgroup generated by the elements

$$
A_{1, i}, A_{2, i}, \ldots, A_{i-1, i}, A_{i, i+1}, \ldots, A_{\text {in }},
$$

where $A_{i, j}$ are those generators of $P_{n}$. Recall that the image of $A_{i j}^{-1}$ under the reduced Gassner representation is denoted by $Y_{i j}$, where $Y_{i j}=I-\overline{P_{i j}} \overline{Q_{i j}}$.

In other words, the generators of $V_{i}$ are $A_{i, j}$ where $A_{i, j}=A_{j, i}$ whenever $i>j$ and $j=1,2, \ldots, n$. It is known that $V_{i}$ generates a free subgroup of $P_{n}$ which is isomorphic to the subgroup $V_{n}$ freely generated by $\left\{A_{1, n}, A_{2, n}, \ldots, A_{n-1, n}\right\}$. This is intuitively clear because it is quite arbitrary how we assign indices to the braid "strings".

Let $\overline{\gamma_{n}}: V_{i} \rightarrow G L_{n-1}\left(\mathbb{Z}\left[y_{1}^{ \pm 1}, \ldots, y_{n}^{ \pm 1}\right]\right)$ and $Y_{i j}$ be the image of the generator $A_{i j}^{-1}$ under $\overline{\gamma_{n}}$. Then

$$
Y_{i j}=I-\overline{P_{i j}} \overline{Q_{i j}},
$$

where $\overline{Q_{i j}} \overline{P_{i k}}=Q_{i j} P_{i k}$.

Again all choices of $\overline{P_{i j}}, \overline{Q_{i j}}$, for which $\left(\overline{Q_{i j}} \overline{P_{i k}}\right)=\left(Q_{i j} P_{i k}\right)$, give equivalent representations of $V_{i}$. Here $P_{k i}=P_{i k}$ and $\overline{P_{k i}}=\overline{P_{i k}}$ whenever $k>i$, and similarly for $Q_{i j}$ and $\overline{Q_{i j}}$. 
Direct calculations show that for $1 \leq i, j, k \leq n$, the matrix $\left(Q_{i j} P_{i k}\right)$ given by the inner product is the following:

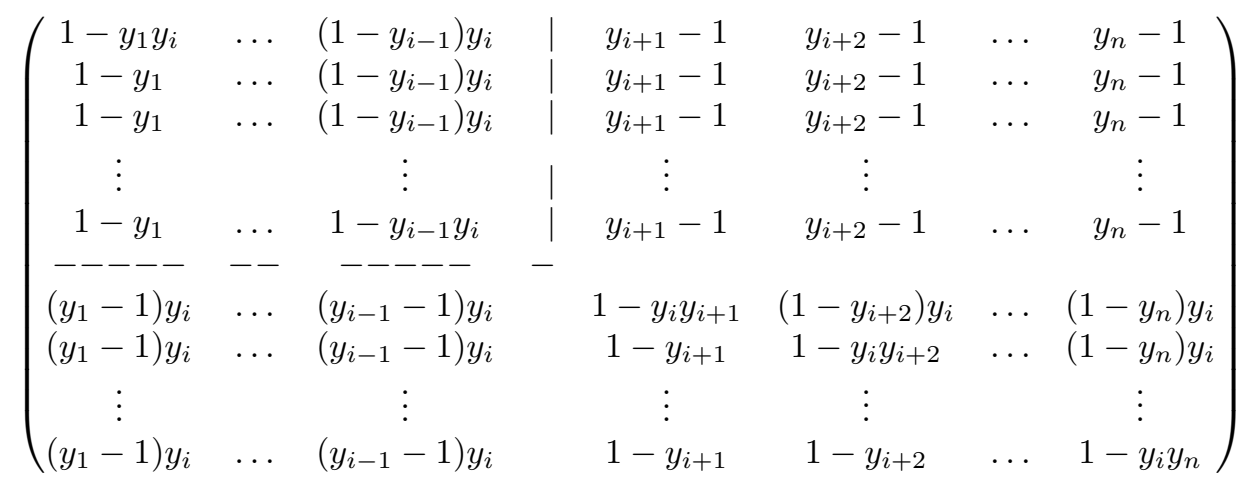

The upper left submatrix is an $(i-1) \times(i-1)$ matrix. More precisely, it is the matrix given by the inner product $\left(Q_{j i} P_{k i}\right)$, where $1 \leq j, k \leq i-1$.

Lemma 6. Let $X_{n}$ be the $(n-1) \times(n-1)$ matrix $\left(Q_{i j} P_{i k}\right)$. Then

$$
\operatorname{det}\left(X_{n}\right)=\left(1-y_{i}\right)^{n-2}\left(1-y_{1} y_{2} \ldots y_{n}\right) .
$$

Theorem 7. Let $i$ be an integer such that $1 \leq i \leq n$. Then $\overline{\gamma_{n}}$ is irreducible on $V_{i}$ if and only if $\left(1-y_{i}\right)^{n-2}\left(1-y_{1} \ldots y_{n}\right) \neq 0$.

Proof. This directly follows by Lemma 6 and Theorem 2 (a).

By Theorem 7, the reduced Gassner representation of $P_{n}$ is irreducible. Recall that the indeterminates involved in defining this representation are independent and that irreducibility of a representation on a subgroup implies the irreducibility on the group itself.

Let $G_{n}(z)$ be the representation

$$
P_{n} \rightarrow G L_{n-1}(\mathbb{C})
$$

obtained by specializing $y_{i} \rightarrow z_{i}$ in the reduced Gassner representation $\overline{\gamma_{n}}$, where $z=\left(z_{1}, \ldots, z_{n}\right) ; z_{1}, \ldots, z_{n} \in \mathbb{C}^{*}$.

Next in my work, I will consider $G L_{n-1}(\mathbb{C})$ acting from the right on row vectors. I will show that if $z=\left(z_{1}, \ldots, z_{n}\right)$ and $z_{1} \ldots z_{n}=1$, then there is a nonzero vector fixed by the specialization of the reduced Gassner representation $G_{n}(z)$.

Proposition 8. If $z_{1} \ldots z_{n}=1$, then the vector $\left[z_{2} \ldots z_{n-1}\left(z_{1}-1\right), z_{3} \ldots\right.$ $\left.z_{n-1}\left(z_{2}-1\right), \ldots, z_{n-1}-1\right]$ is fixed by $G_{n}(z)$.

Proof. For simplicity, denote the above vector by $v$ and for $i=1,2, \ldots n-2$ define $a_{i}$ and $a_{n-1}$ as follows:

$$
a_{i}=z_{i+1} \ldots z_{n-1}\left(z_{i}-1\right) \quad \text { and } \quad a_{n-1}=z_{n-1}-1 .
$$

If $z_{i}=1$ for every $i=1, \ldots, n-1$, then it is clear that, by our hypothesis, $z_{n}=1$. In this case, $G_{n}(z)$ will be trivial. Therefore, we may assume that $z_{i} \neq 1$ for some $i=1, \ldots, n-1$.

Consider here $Y_{i j}$ as the image of $A_{i j}^{-1}$ under the complex specialization of the reduced Gassner representation of $P_{n}$. I will show that for every $1 \leq i<j \leq n$, we 
have

$$
v Y_{i j}=v
$$

Case 1. Consider $Y_{i j}$ for $j \neq n$,

$$
Y_{i j}=I-\overline{P_{i j}} \overline{Q_{i j}}
$$

where

$\overline{P_{i j}}=$

$(\underbrace{0 \ldots 0}_{i-1} \quad z_{j}-1 \quad\left(z_{i}-1\right)\left(1-z_{j}\right) \ldots \quad\left(z_{i}-1\right)\left(1-z_{j}\right) \quad\left(z_{i}-1\right)\left(-z_{j}\right) \quad \underbrace{0 \ldots 0}_{n-1-j})^{T}$

and

$$
\begin{gathered}
\overline{Q_{i j}}=\left(\begin{array}{cccccc}
\underbrace{0 \ldots 0}_{i-1} & -1 & 0 \ldots & 0 & 1 & \underbrace{0 \ldots 0}_{n-1-j}
\end{array}\right) . \\
v Y_{i j}=v\left(I-\overline{P_{i j}} \overline{Q_{i j}}\right)=v-\left(v \overline{P_{i j}}\right) \overline{Q_{i j}} .
\end{gathered}
$$

Simple calculation shows that $v \overline{P_{i j}}=\left(1-z_{j}\right)\left(z_{i}-1\right) A$, where

$$
\begin{aligned}
A= & -z_{i+1} \ldots z_{n-1}+z_{i+1} \ldots z_{n-1} \\
& -z_{i+2} \ldots z_{n-1}+z_{i+2} \ldots z_{n-1} \\
& \vdots \\
& -z_{j} \ldots z_{n-1}+z_{j} \ldots z_{n-1} .
\end{aligned}
$$

Simplifying, we obtain that

$$
A=0 \text {. }
$$

By (1), we get

$$
v Y_{i j}=v .
$$

Case 2. Let $j=n$ and consider $Y_{i n}=I-\overline{P_{i n}} \overline{Q_{i n}}$, where

$$
\overline{P_{\text {in }}}=(\underbrace{\left(1-z_{i}\right) z_{n} \ldots\left(1-z_{i}\right) z_{n}}_{i-1} 1-z_{i} z_{n} \underbrace{1-z_{i} \ldots 1-z_{i}}_{n-1-i})^{T}
$$

and

$$
\overline{Q_{i n}}=\left(\begin{array}{lll}
\underbrace{0 \ldots 0}_{i-1} & 1 & \underbrace{0 \ldots 0}_{n-1-i}
\end{array}\right) .
$$

Again

$$
v Y_{i n}=v-\left(v \overline{P_{i n}}\right) \overline{Q_{i n}} .
$$


By similar calculations as in case 1 , we get $v \overline{P_{i n}}=\left(1-z_{i}\right) B$, where

$$
\begin{aligned}
B= & z_{1} \ldots z_{n-1} z_{n}-z_{2} \ldots z_{n-1} z_{n} \\
& +z_{2} \ldots z_{n-1} z_{n}-z_{3} \ldots z_{n-1} z_{n} \\
& \vdots \\
& -z_{i+1} \ldots z_{n-1}+z_{i} \ldots z_{n} \\
& +z_{i+1} \ldots z_{n-1}-z_{i+2} \ldots z_{n-1} \\
& \vdots \\
& +z_{n-1}-1
\end{aligned}
$$

Since, by our hypothesis, $z_{1} \ldots z_{n}=1$, it follows that, after simplifying,

$$
B=0 .
$$

Hence by (2), we get $v Y_{\text {in }}=v$.

Therefore, by treating all the possible cases, we have proved that for $1 \leq i<$ $j \leq n$

$$
v Y_{i j}=v
$$

Recall that irreducibility of a representation of a group in $G L_{n-1}(\mathbb{C})$ means that there are no invariant subspaces under the action of this representation. Now we present the proof of the main theorem.

\section{Proof of Main Theorem}

Suppose, to get a contradiction, that $z_{1} \ldots z_{n}=1$. Then, by Proposition 8 , there is a nonzero vector $v$ which is fixed by $G_{n}(z)$. This contradicts the irreducibility of $G_{n}(z)$.

Now assume that $z_{1} \ldots z_{n} \neq 1$. I will show that $G_{n}(z)$ is irreducible. Since $z_{1} \ldots z_{n} \neq 1$, it follows that at least one $z_{i} \neq 1$ for some $i=1, \ldots, n$. Then we have

$$
\left(1-z_{i}\right)^{n-2}\left(1-z_{1} \ldots z_{n}\right) \neq 0 \text {. }
$$

Considering the corresponding subgroup $V_{i}$, we find out that $G_{n}(z)$ is irreducible on $V_{i}$ by Theorem 7 . Hence irreducibility on $P_{n}$ follows immediately.

\section{REFERENCES}

[1] M. Abdulrahim, The Gassner Representation of the Pure Braid Group, Doctoral Thesis, The Pennsylvania State University, 1995.

[2] J.S. Birman, Braids, Links and Mapping Class Groups, Vol 82 of Annals of Mathematical Studies, Princeton University Press, New Jersy, 1975. MR 51:11477; MR 54:13894

[3] L. Dornhoff, Group Representation Theory, Part A, Marcel Dekker Inc., New York, 1971. MR 50:458a

[4] E. Formanek, Braid group representations of low degree, Proc. London Math. Soc. 73 (1996), 279-322. CMP 96:15

[5] V.L. Hansen, Braids and Coverings, London Mathematical Society. Cambridge University Press, 1989. MR 94g:57004

Department of Mathematics, The Pennsylvania State University, University Park, Pennsylvania 16802

E-mail address: mna@math.psu.edu 